\title{
Co-occurring hydrocephalus in autism spectrum disorder: a Danish population- based cohort study
}

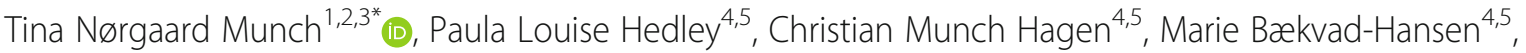
Jonas Bybjerg-Grauholm ${ }^{4,5}$, Jakob Grove ${ }^{5,6,7,8}$, Merete Nordentoft, ${ }^{5,9}$, Anders Dupont Børglum ${ }^{5,9,10}$, Preben Bo Mortensen ${ }^{5,11}$, Thomas Mears Werge ${ }^{5,9}$, Mads Melbye ${ }^{12}$, David Michael Hougaard ${ }^{4,5}$ and Michael Christiansen ${ }^{4,5,13}$

\begin{abstract}
Background: The association between autism spectrum disorder and hydrocephalus is not well understood, despite demonstrated links between autism spectrum disorder and cerebrospinal fluid abnormalities. Based on the hypothesis that autism spectrum disorder and hydrocephalus may, at least in some cases, be two manifestations of a shared congenital brain pathology, we investigated the potential association between autism spectrum disorder and hydrocephalus in a large Danish population-based cohort.

Methods: Patients and controls were obtained from the Lundbeck Foundation Initiative for Integrative Psychiatric Research iPSYCH2012 case-cohort, which includes all patients with selected psychiatric disorders born in Denmark 1981-2005 along with randomly selected population controls (end of follow-up, December 31, 2016). The associations between individual psychiatric disorders and hydrocephalus were estimated using binary logistic regression with adjustment for age and sex.

Results: The cohort consisted of 86,571 individuals, of which 14,654 were diagnosed with autism spectrum disorder, 28,606 were population controls, and the remaining were diagnosed with other psychiatric disorders. We identified 201 hydrocephalus cases; 68 among autism spectrum disorder patients and 40 among controls (OR 3.77, 95\% Cl 2.48-5.78), which corresponds to an absolute risk of $0.46 \%$ (i.e. approximately one in 217 children with autism spectrum disorder had co-occurring hydrocephalus). The autism spectrum disorder-hydrocephalus association was significant over the entire subgroup spectrum of autism spectrum disorder.

\footnotetext{
* Correspondence: tina.noergaard.munch@regionh.dk

'Department of Epidemiology Research, Statens Serum Institut, Copenhagen, Denmark

${ }^{2}$ Department of Neurosurgery, Copenhagen University Hospital, Blegdamsvej 9, DK-2100 Copenhagen, Denmark

Full list of author information is available at the end of the article
}

C C The Author(s). 2021 Open Access This article is licensed under a Creative Commons Attribution 4.0 International License, which permits use, sharing, adaptation, distribution and reproduction in any medium or format, as long as you give appropriate credit to the original author(s) and the source, provide a link to the Creative Commons licence, and indicate if changes were made. The images or other third party material in this article are included in the article's Creative Commons licence, unless indicated otherwise in a credit line to the material. If material is not included in the article's Creative Commons licence and your intended use is not permitted by statutory regulation or exceeds the permitted use, you will need to obtain permission directly from the copyright holder. To view a copy of this licence, visit http://creativecommons.org/licenses/by/4.0/. The Creative Commons Public Domain Dedication waiver (http://creativecommons.org/publicdomain/zero/1.0/) applies to the data made available in this article, unless otherwise stated in a credit line to the data. 


\begin{abstract}
(Continued from previous page)
Conclusions: Given the considerable risk of hydrocephalus among patients with autism spectrum disorder, we suggest that patients with autism spectrum disorder should be evaluated for co-occurring hydrocephalus on a routine basis as timely neurosurgical intervention is important. Likewise, attention must be paid to traits of autism spectrum disorder in children with hydrocephalus. The results of this study call for future investigations on a potential shared aetiology between hydrocephalus and autism spectrum disorder, including the role abnormal CSF dynamics in the pathogenesis of autism spectrum disorder.
\end{abstract}

Keywords: Autism spectrum disorder, Hydrocephalus, Epidemiology, Cohort, Congenital

\section{Background}

In Denmark, the setting for this study, the prevalence of autism spectrum disorder in children is reported to be $68.5 / 10,000$ [1] with a male to female ratio of 4:1 [2]. Characterized by impairments in social interaction, communication, and repetitive or restrictive behaviors [3], autism spectrum disorder is associated with a plethora of comorbidities, often neurodevelopmental in nature $[4,5]$. Shen et al. showed in different MRI studies that children with autism spectrum disorder, up to preschool age, consistently exhibit increased volumes of CSF in the subarachnoid space, as compared to other children, a condition also known as external hydrocephalus [6-9].

Hydrocephalus is an aetiologically heterogeneous neurological condition with the broad definition excessive amount of intracranial CSF relative to the brain volume, which occurs in 1.08/1,000 children up to 2 years of age in Denmark [10]. A relatively common presentation is the Benign Enlargement of the Subarachnoid Space (BESS) or benign external hydrocephalus, that is asymptomatic and usually resolves spontaneously before the child reaches the age of 2 years $[11,12]$. The signs and symptoms which most hydrocephalus patients present with do not necessarily include the well-known acute symptoms of increased intracranial pressure or obvious increased head circumference. They may present with normal or only slightly increased head circumference, and variable degrees of psycho-motoric delay or failure to thrive during infancy. When neurosurgical treatment is required, they undergo endoscopic fenestration of the third ventricle or insertion of a ventriculoperitoneal shunt.

Although children with hydrocephalus needing surgical treatment remarkedly often display traits compatible with autism spectrum disorder, only one population-based study has investigated this potential association. Thus, Lindquist et al. reported that autism was present in nine out of the 107 children with hydrocephalus (13\%) born in western Sweden during the period 1989-1993 [13]. However, this potential association has not been investigated in larger cohorts before. Most likely, this is due to the relatively low prevalence of both diseases, which would require very large study populations. The access to the unique iPSYCH cohort data enabled us to investigate this question in a large population-based cohort including 14, $000+$ individuals with autism spectrum disorder.

Based on the hypothesis that autism spectrum disorder and hydrocephalus may, at least in some cases, be two manifestations of a shared congenital brain pathology, we investigate (1) the association between autism spectrum disorder and hydrocephalus in a large Danish population-based cohort and (2) the associations between hydrocephalus and the psychiatric disorders: schizophrenia, bipolar disorder, major depressive disorder, autism spectrum disorder, and attention-deficit hyperactivity disorder (ADHD).

\section{Methods}

\section{Data sources}

This register-based case-cohort study is a sub-study of the Lundbeck Foundation Initiative For Integrative Psychiatric Research (iPSYCH, http:www.iPSYCH.au.dk) and combines data from The National Patient Register [14], The Danish Neonatal Screening Biobank [15], and the Danish Psychiatric Central Research Register [16]. The registers contains information about all in-patient admissions since 1969 and out-patient visits from 1995 onwards. The data were linked on an individual level using the unique personal identification number from the Danish Civil Registration System [17].

\section{Cohort}

Patients and controls were obtained from the Lundbeck Foundation Initiative for Integrative Psychiatric Research iPSYCH2012 case-cohort [18], which comprises information on individuals with psychiatric disorders $(n=57$, $875)$ as well as a randomly sampled control cohort from the same source population as the psychiatric cases $(n=$ 28,606). Thus, the controls represent the background population. Cases and the population cohort were selected from a study base of 1,472,762 singleton births between May 1, 1981, and December 31, 2005, who were alive and resided in Denmark at their first birthday. End of follow-up was December 31, 2016. Hence, the cohort members were followed for at least 11 years and up to 34 years and 8 months. Cases were defined as all individuals registered with a diagnosis of five selected psychiatric disorders: schizophrenia, bipolar disorder, major 
depressive disorder, autism spectrum disorder, and attention-deficit and hyperactivity disorder.

\section{Variables}

The outcome variables were a registered diagnosis of at least one of the psychiatric disorders and/or a registered diagnosis of hydrocephalus. The ICD-8 system was used from 1981 to 1994 , but it was replaced by the ICD-10 system from 1995 and onwards.

\section{Ascertainment of psychiatric disease}

The study base was linked, through the personal identification number, to the Danish Psychiatric Central Research Register to obtain information about psychiatric disorders, defined by the ICD-10 codes: F20 schizophrenia, F30-F31 bipolar disorder, F32-F39 major depressive disorder, F84 autism spectrum disorder, or F90 attention-deficit hyperactivity disorder (ADHD). ASD was furthermore divided into the subgroups defined in the ICD-10 system: F84.0 childhood autism, F84.1 atypical autism, F84.5 Asperger's syndrome, F84.8 other pervasive developmental disorder, and F84.9 pervasive developmental disorder, unspecified. The ASD diagnosis in the Danish Psychiatric Central Register has been validated [19].

In cases where more than one condition was diagnosed, the diagnoses were prioritized with respect to their rank in the ICD-10 code system. Thus, for this study, each individual was only captured within one diagnostic category, according to the following order: schizophrenia $>$ bipolar disorder $>$ major depressive disorder $>$ autism spectrum disorder $>$ attention-deficit and hyperactivity disorder. Therefore, the case numbers of the psychiatric disorders are somewhat different from the case numbers of the iPSYCH cohort described by Pedersen et al., in which the diagnoses were not ranked, and the cohort members might have more than one diagnosis [18].

\section{Ascertainment of hydrocephalus}

The study base was linked, through the personal identification number, to The National Patient Register. Hydrocephalus cases were identified using the wide range of existing hydrocephalus diagnoses. In case of more than one diagnosis, the following ranking order was used, according to specificity: G91.1 obstructive hydrocephalus; 74200, 74201, 74208, 74209, Q038x, and Q039 congenital hydrocephalus; G91.0 communicating hydrocephalus; G91.3, 34794 post traumatic and acquired hydrocephalus; and G91.8, G91.9 hydrocephalus, not specified. As the ICD-10 system is the most recent and most specific classification used during the study period, the ICD-10 diagnoses were ranked higher than the ICD-8 diagnoses.

We deliberately did not include hydrocephalus related to birth trauma (P10x, P91.7) and spontaneous perinatal intracranial haemorrhage (P52), because they have such obvious acquired aetiologies. We chose to include post traumatic hydrocephalus because hydrocephalus/ventriculomegaly is a relatively common incidental finding on brain CT scans performed at emergency units in patients who suffered mild to moderate head trauma. Thus, incidental findings of subclinical congenital hydrocephalus may be interpreted as post traumatic hydrocephalus. Only few patients actually develop symptomatic hydrocephalus after severe head trauma (3.56\%) [20], and those who do may hold genetic predisposing factors.

\section{Statistical analysis}

The risk of hydrocephalus among patients with psychiatric disorders compared to background population controls were estimated as odds ratios (ORs) using logistic regression analysis including age and sex as covariates, both in the primary analyses and sub-analyses. A proportion test was performed to test for differences between groups with the autism spectrum disorder-hydrocephalus association as reference. A $p$-value of 0.05 was considered significant and Bonferroni correction for multiple testing was performed where appropriate. For groupings with 30+ hydrocephalus-positive cases, a sampling distribution of the test statistic of the logit model of the hydrocephalus status was resampled $25 \mathrm{~K}$ times and the coefficients for hydrocephalus extracted from the logit model and compared to the observed. The $p$-values were computed as two-sided. As the number of hydrocephalus-positive observations within the groupings was relatively low, the statistical tests were confirmed by a Fisher's exact test (data not shown). Wilcoxon rank sum test was used to compare median age at diagnosis of hydrocephalus among controls and each of the psychiatric disorders.

\section{Results}

Basic characteristics of the cohort are presented in Table 1, including the numbers of patients with each of the five psychiatric disorders, median age at diagnosis, and by the end of follow-up. Among the 86,481 cohort members, we identified 201 hydrocephalus cases, distributed as follows: 40 among the 28,606 population controls, 68 among the 14, 654 patients with autism spectrum disorder (OR 3.77, 95\% CI $2.48-5.78, p \leq 4 \times 10^{-5}$, and 37 among the 13,901 patients with ADHD (OR 1.91, 95\% CI 1.20-3.04, $p=0.07$ ), as presented in Table 2 . The remaining 56 cohort members with hydrocephalus were diagnosed with either major depressive disorder, bipolar disorder, or schizophrenia, but no significant associations were found between hydrocephalus and these disorders. Nevertheless, the OR for hydrocephalus among the 5229 cohort members with schizophrenia was as high as $2.01,95 \%$ CI 1.00-3.83, $p=$ 0.51 so this association may have reached statistical significance with higher numbers. The strong association 
Table 1 Basic characteristics of the study population

\begin{tabular}{lllll}
\hline & $\mathbf{N}$ & $\begin{array}{l}\text { Median age in years, Dec } \mathbf{2 0 1 6}^{\mathbf{b}} \\
\text { (interquartile range) }\end{array}$ & $\begin{array}{l}\text { Male } \\
\text { (\%) }\end{array}$ & $\begin{array}{l}\text { Median age at diagnosis in years } \\
\text { (interquartile range) }\end{array}$ \\
\hline No psychiatric disease (controls) & 28,606 & $22.6(16.9-28.6)$ & 50.4 & Not relevant \\
Schizophrenia & 5242 & $28.6(25.3-32.2)$ & 53.3 & $21.2(18.9-24.0)$ \\
Major depressive disorder & 22,868 & $28.8(25.0-32.2)$ & 31.9 & $20.0(17.0-23.3)$ \\
Bipolar disorder & 2300 & $29.7(26.5-32.8)$ & 35.6 & $22.9(20.0-26.5)$ \\
Autism spectrum disorder & 14,564 & $19.8(16.2-23.9)$ & 80.9 & $9.6(6.4-13.3)$ \\
$\quad \begin{array}{l}\text { Childhood autism } \\
\text { Atypical autism }\end{array}$ & 4447 & $17.9(14.6-21.6)$ & 81.4 & $7.3(4.8-12)$ \\
$\quad \begin{array}{l}\text { Asperger's syndrome } \\
\text { Other PDD }\end{array}$ & 1803 & $19.7(16.2-23.6)$ & 75.8 & $10.8(7.1-14.4)$ \\
$\quad \begin{array}{l}\text { Unspecified PDD } \\
\text { Attention-deficit hyperactivity }\end{array}$ & 18405 & $21.7(17.7-25.9)$ & 83.6 & $11.5(8.6-14.9)$ \\
disorder & 12,901 & $21.2(16.8-25.7)$ & 80.9 & $10.5(7.6-13.5)$ \\
\hline
\end{tabular}

${ }^{\mathrm{a} P e r v a s i v e}$ developmental disorder.

${ }^{b}$ End of follow-up

between hydrocephalus and autism spectrum disorder was supported by permutation testing (Supplementary figure 1). Additionally, we performed a proportion test, as presented in Table 2. The results supported that the autism spectrum disorder-hydrocephalus association was significantly different from the associations between the other psychiatric disorders and hydrocephalus, although the schizophrenia-hydrocephalus association was close to not being significantly different $(p=0.047)$.

Furthermore, we investigated the associations between each of the clinical subgroups of autism spectrum disorder and hydrocephalus, as presented in Table 3. In all five subgroups, we found a significant association with hydrocephalus: childhood autism (OR 3.71, 95\% CI 2.08-6.51), atypical autism (OR 5.58, 95\% CI 2.81-10.4), Asperger's syndrome (OR 2.88, 95\% CI 1.49-5.31), other pervasive developmental disorders (OR 3.35, 95\% CI 1.41-7.02), and pervasive developmental disorders, unspecified (OR 3.46, 95\% CI 1.53-7.01).

\section{Sub-analyses}

In a sub-analysis, we subdivided the ICD-codes for hydrocephalus into five groups to see if the association between ASD and hydrocephalus was driven by a particular subgroup of hydrocephalus. The results are presented in Table 4. Only the subgroups obstructive hydrocephalus $(\mathrm{OH})$ and congenital hydrocephalus $(\mathrm{CH})$ reached sufficient numbers for estimating odd's ratios. The results showed that the associations between autism spectrum disorder and both of the groups were significant; OR ASD-OH 5.89, 95\% CI 3.02-12.10 ( $p<4.0 \mathrm{E}-5)$, and OR ASD-CH 2.50, 95\% CI 1.30-4.84 ( $p=0.0067)$.

The diagnostic process and the diagnostic definition of autism spectrum disorder changed significantly during the period 1981-2005, in which the cohort members were born. This meant that cohort members diagnosed with autism spectrum disorder before 1994 were not registered with an autism spectrum disorder diagnosis before the implementation of the ICD-10 system in

Table 2 Associations between hydrocephalus and five psychiatric disorders

\begin{tabular}{|c|c|c|c|c|c|c|}
\hline \multirow[t]{2}{*}{ Condition } & \multicolumn{2}{|c|}{$\begin{array}{l}\text { Hydrocephalus }(N= \\
\text { 201) }\end{array}$} & \multicolumn{3}{|c|}{$\begin{array}{l}\text { Logistic regression adjusted for age } \\
\text { and sex }\end{array}$} & \multirow{2}{*}{$\begin{array}{l}\text { Proportion test between groups } \\
p \text {-value }\end{array}$} \\
\hline & Present $N$ & Absent $N$ & OR & $95 \% \mathrm{Cl}$ & $p$-value ${ }^{a}$ & \\
\hline No psychiatric disease (controls) & 40 & 28,566 & 1 & Reference & Reference & - \\
\hline Schizophrenia & 13 & 5229 & 2.01 & $(1.00-3.83)$ & 0.5 & 0.047 \\
\hline Major depressive disorder & 40 & 22,828 & 1.22 & $(0.75-1.99)$ & 1.0 & $<5 \times 10^{-7}$ \\
\hline Bipolar disorder & 3 & 2297 & 1.06 & $(0.25-3.08)$ & 1.0 & 0.03 \\
\hline Autism spectrum disorder & 68 & 14,586 & 3.77 & $(2.48-5.78)$ & $<4 \times 10^{-5}$ & Reference \\
\hline Attention-deficit and hyperactivity disorder & 37 & 12,864 & 1.91 & $(1.20-3.04)$ & 0.07 & 0.02 \\
\hline
\end{tabular}

${ }^{\mathrm{a} B o n f e r r o n i}$ corrected for multiple tests (here five) 
Table 3 Associations between hydrocephalus and clinical subgroups of autism spectrum disorders

\begin{tabular}{|c|c|c|c|c|c|}
\hline \multirow[t]{2}{*}{ Condition } & \multicolumn{2}{|c|}{ Hydrocephalus } & \multicolumn{3}{|c|}{ Logistic regression adjusted for age and sex } \\
\hline & Present $N$ & Absent $N$ & $\overline{\mathrm{OR}}$ & $(95 \% \mathrm{Cl})$ & $p$-value ${ }^{a}$ \\
\hline No psychiatric disease (controls) & 40 & 28,566 & 1 & Reference & Reference \\
\hline Childhood autism & 23 & 4424 & 3.71 & $(2.08-6.51)$ & $3 \times 10^{-5}$ \\
\hline Atypical autism & 13 & 1790 & 5.58 & $(2.81-0.40)$ & $1.0 \times 10^{-6}$ \\
\hline Asperger's syndrome & 15 & 4390 & 2.88 & $(1.49-5.31)$ & 0.005 \\
\hline Other pervasive developmental disorder & 8 & 1860 & 3.35 & $(1.41-7.02)$ & 0.014 \\
\hline Pervasive developmental disorder, unspecified & 9 & 2122 & 3.46 & $(1.53-7.01)$ & 0.006 \\
\hline
\end{tabular}

${ }^{a}$ Bonferroni corrected for multiple tests (here five)

1995. The median age at diagnosis for all cohort members with autism spectrum disorder by year of birth are presented in Fig. 1. The decreasing age at diagnosis during the time period supports this notion.

A description of age at diagnosis and gender distribution of the hydrocephalus patients by co-occurring psychiatric disorders compared to hydrocephalus patients without psychiatric disorders are presented in Supplementary Table 1 . Only patients with schizophrenia and major depressive disorder were diagnosed with hydrocephalus significantly later than the controls without cooccurring psychiatric disorders. Thus, hydrocephalus patients with autism spectrum disorder were diagnosed with hydrocephalus at a median age of 1.0 year (IQR $0.3-5.3$ years), which was not significantly different from the median age of hydrocephalus diagnosis among controls without psychiatric disorders $(2.3$ years, IQR $0.3-$ 12.1, $P=0.4$ ).

The sequence of diagnoses in the 68 individuals with both autism spectrum disorder and hydrocephalus, as well as the subgroup of 23 individuals diagnosed with "childhood autism", which is the autism spectrum disorder sub diagnosis presenting at the youngest age, is presented in Supplementary figure 2. The diagnosis of hydrocephalus preceded that of autism spectrum disorder in 55 cases $(81 \%)$, whereas autism spectrum disorder preceded hydrocephalus in 13 cases (19\%). A similar pattern was seen in the subgroup of 23 individuals with childhood autism and hydrocephalus. However, the results presented in Supplementary figure 2 must be interpreted with caution, because the sequence of diagnoses does not necessarily reflect the biological sequence of the diseases due to the delays of autism spectrum disorder diagnoses, as described above and in Fig. 1.

\section{Discussion}

This is the first demonstration of a strong co-occurrence of autism spectrum disorder and hydrocephalus in a large population-based cohort (OR 3.77, 95\% CI 2.485.78). This association was significant for the entire subgroup spectrum of autism spectrum disorder. The absolute risk of hydrocephalus among children with autism spectrum disorder was $0.46 \%$ (i.e., approximately one in 217 children with autism spectrum disorder had cooccurring hydrocephalus).

The observed strong association between autism spectrum disorder and hydrocephalus is in line with the findings of the, to our knowledge, only other population-based investigation of the subject matter. Thus, Lindquist et al. reported that nine out of the 107 children born with hydrocephalus in western Sweden during the period 1989-1993 were diagnosed with autism (13\%) [13]. The occurrence of autism spectrum disorder among non-myelomeningocele hydrocephalus patients was as high as $20 \%$, when actually evaluated as part of the study protocol using the Childhood Autism Rating Scale [21].

It is well known that autism spectrum disorder exhibits considerable psychiatric comorbidity, most notably

Table 4 Median age at hydrocephalus diagnosis, and association with autism spectrum disorder, according to ICD-subtype distribution of hydrocephalus

\begin{tabular}{|c|c|c|c|c|}
\hline Hydrocephalus diagnoses (ICD-8 and ICD-10 codes) $^{\mathrm{a}}$ & $N(201)$ & Median age at diagnosis & OR $95 \% \mathrm{Cl}$ & $P$-value \\
\hline Obstructive hydrocephalus ${ }^{\mathrm{b}}$ (G911) & 67 & 8.3 & $5.89(3.02-12.10)$ & $<4.0 \mathrm{E}-5$ \\
\hline Communicating hydrocephalus $^{\mathrm{b}}$ (G910, G912) & 17 & 18.4 & - & - \\
\hline Congenital hydrocephalus (74200, 74201, 74208, 74209, Q038x, Q039) & 79 & 0.8 & $2.50(1.30-4.84)$ & 0.0067 \\
\hline Post traumatic/acquired hydrocephalus $(34794$, G913) & 7 & 2.3 & - & - \\
\hline Hydrocephalus, not specified ${ }^{\mathrm{b}}$ (G918, G919) & 31 & 2.7 & - & - \\
\hline
\end{tabular}

The more recent and more specific ICD-10 system diagnoses was ranked than the ICD-8 diagnoses

an case of more than one hydrocephalus diagnosis, the more specific diagnosis was ranking higher

${ }^{b}$ Congenital $(\mathrm{Q})$, or likely to be congenital due to the low median age at hydrocephalus diagnosis and the young age of the study population (G) 


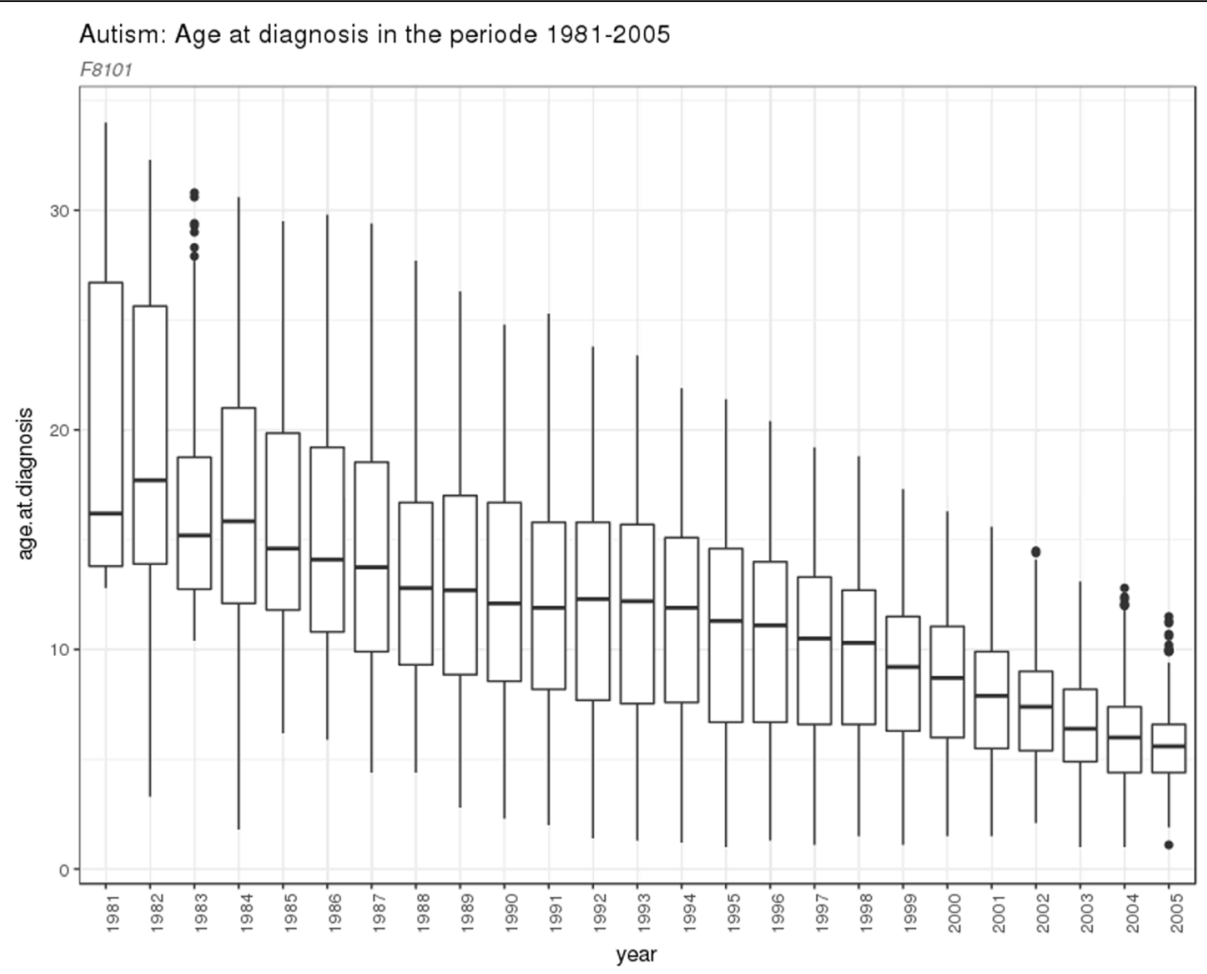

Fig. 1 The median age at diagnosis for all cohort members with autism spectrum disorder by year of birth

with other neurodevelopmental disorders [4]. A total of 3625 of the cohort members with autism spectrum disorder in this study had co-occurring ADHD (25\%), and a borderline significant association between ADHD and hydrocephalus was observed. A large-scale genetic study published in Science 2018 found evidence of a shared heritability between different psychiatric disorders, which may explain the frequently observed cooccurrence with ADHD [22].

It has previously been reported that co-occurring neurological or neurodevelopmental diagnoses are associated with later diagnosis of autism spectrum disorder $[23,24]$. According to these studies, the presence of psychiatric and neurological disorders may result in masking or obscuring of core symptoms, which leads to delayed autism spectrum disorder diagnosis. Another reason can be high functioning level, as in Asperger, which may lead to later diagnosis. This may partly explain our finding of a relatively high median age for diagnosis of autism spectrum disorder diagnosis of 9.6 years. This notion is supported by the fact that the number of years to the second diagnosis was considerably longer if hydrocephalus was diagnosed first (Supplementary figure 2). Another important previously mentioned reason for the high median age is the delay of registered diagnoses of neurodevelopmental disorders for the cohort members born before the change into the ICD-10 system in 1995. This issue is addressed in the second of the sub analyses. As presented in Fig. 1, the median age at diagnosis decreased over the birth years of the cohort members from 1981 to 2015 . Hence, the historical median age of diagnosis of autism spectrum disorders of 9.6 years observed in this study is not directly comparable to recent observations.

Our finding of a strong association between autism spectrum disorder and hydrocephalus may indicate either a causal association or a shared aetiology. Previous studies indicate that abnormal CSF dynamics may play a role in the development of autism spectrum disorder. Thus, Shen et al. demonstrated increased amounts of extra-axial CSF in children with autism spectrum disorder and age from 6 months to 4 years of age, regardless of familial risk [6-9]. Shen et al. concluded that "it is not clear whether increased CSF in the subarachnoid space may directly impact brain development and contribute to the pathology of psychiatric disorders such as ASD, or is an epiphenomenon that reflects another underlying aetiology" [8]. Either way, these findings, together with the strong association observed in this study, pave the way for the hypothesis that autism spectrum disorder could be associated with hydrocephalus through disturbed CSF dynamics, perhaps through shared genetic factors.

One could argue that the association observed in this study between autism spectrum disorder and hydrocephalus could also rely on a direct causal association, meaning that patients with hydrocephalus may develop autism 
spectrum disorder symptoms due to the brain damage caused by pressure to the periventricular structures in the brain. We cannot completely reject a causal association, but we do find it unlikely given the fact that even though the absolute risk of $0.46 \%$ is considerable from a clinical perspective, we would expect a higher proportion to be affected in case of a direct causal association.

Interestingly, we also observed a substantial number of hydrocephalus cases among patients with schizophrenia $(0.25 \%)$. In fact, mild ventriculomegaly is considered a hallmark feature in particularly early onset schizophrenia together with other structural brain abnormalities indicating a neurodevelopmental aetiology [25-27]. In the elderly, schizophrenia may be associated with normal pressure hydrocephalus $[28,29]$.

A potential limitation of this study is the potential of surveillance bias, e.g. patients with severe psychiatric disorders may have a higher probability of undergoing brain imaging studies than background population controls and therefore incidental findings of hydrocephalus could be more likely in these patients. However, the significant differences between the association between autism spectrum disorder and hydrocephalus and the associations between hydrocephalus and the other psychiatric disorders, as presented in Table 2, argue against surveillance bias. However, one could argue that patients with autism spectrum disorder are more likely to undergo brain imaging studies than patients with the other psychiatric disorders investigated in this study. But the very strong association observed in this study (OR $3.77,95 \%$ CI $2.48-5.78$ ) is unlikely to be explained by incidental findings of hydrocephalus only, which generally is a rare disease seen in $1.08 / 1000$ in children up to 2 years of age in Denmark [10]. Thus, we believe the effect of surveillance bias has limited impact on the results.

Another limitation is the potential for misclassification of diagnoses in a register-based study. However, autism spectrum disorder and hydrocephalus were the main diagnoses of interest and are two relatively distinct clinical diagnoses, so we believe the misclassification is limited. We deliberately chose to include congenital hydrocephalus and those of the acquired cases that are also considered to have a genetic predisposition, such as post traumatic or post meningitis hydrocephalus cases. We consider that environmental, causative factors, such as head trauma or meningitis, may require the interaction of a genetic factor, in order to manifest acquired hydrocephalus. This would explain why hydrocephalus after head trauma and meningitis is rare, also in our cohort (Table 4). Other common causes for secondary hydrocephalus are subarachnoid haemorrhage from intracranial aneurysms and intracerebral haematoma (stroke), but our cohort members have not yet reached the typical age for stroke as they are only up to 35 years old.
This study has several strengths, the most important is the case-cohort design, which is less prone to the biases of concern in conventional case-control studies because the cohort is randomly selected from the entire population-based birth cohort and therefore representative for the entire Danish background population. The design also minimized loss to follow-up and missing data as reporting of diagnoses for each hospital contact to The National Patient Register is mandatory. The fact that the study is based on nationwide data, not data for a specific region or hospital only, supports the external validity of the results.

\section{Conclusions}

Irrespective of the cause of the association, our findings have clinical consequences as our data suggest that in patients with either disease, the significant risk of the presence of the other should be considered. Thus, patients diagnosed with autism spectrum disorder should be evaluated with imaging studies of the brain on a routine basis and attention must be paid for traits of autism spectrum disorder in children with hydrocephalus. Timely neurosurgical treatment of hydrocephalus when both conditions are present will improve the environment for the per se challenged brain development. The results of this study call for future investigations on a potential shared aetiology between hydrocephalus and autism spectrum disorder, including the role abnormal CSF dynamics in the pathogenesis of autism spectrum disorder.

\section{Abbreviations}

ADHD: Attention-deficit and hyperactivity disorder; CSF: Cerebrospinal fluid; MRI: Magnetic resonance imaging

\section{Supplementary Information}

The online version contains supplementary material available at https://doi. org/10.1186/s11689-021-09367-0.

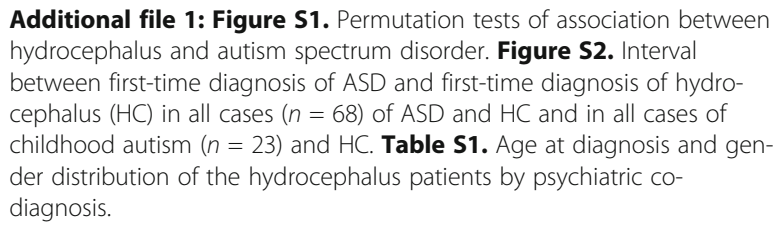

Additional file 1: Figure S1. Permutation tests of association between hydrocephalus and autism spectrum disorder. Figure S2. Interval between first-time diagnosis of ASD and first-time diagnosis of hydrocephalus $(\mathrm{HC})$ in all cases $(n=68)$ of ASD and HC and in all cases of childhood autism $(n=23)$ and HC. Table S1. Age at diagnosis and gender distribution of the hydrocephalus patients by psychiatric codiagnosis.

\section{Acknowledgements}

Not applicable.

\section{Authors' contributions}

Tina N. Munch: study concept and design, study supervision, interpretation of results, writing of the manuscript, critical revision of the manuscript for important intellectual content, and approval of the final manuscript. Paula Hedley: study concept and design, interpretation of results, co-writing of the manuscript, critical revision of the manuscript for important intellectual content, and approval of the final manuscript. Michael Christiansen: study concept and design, study supervision, interpretation of results, co-writing of the manuscript, critical revision of the manuscript for important intellectual content, and approval of the final manuscript. Christian Munch Hagen: 
acquisition of data, statistical analysis, critical revision of the manuscript for important intellectual content, and approval of the final manuscript. Jakob Grove: statistical analysis, critical revision of the manuscript for important intellectual content, and approval of the final manuscript. Anders Børglum: acquisition of data, critical revision of the manuscript for important intellectual content, and approval of the final manuscript. Merethe Nordentoft: acquisition of data, critical revision of the manuscript for important intellectual content, and approval of the final manuscript. Marie Bækvad Hansen: acquisition of data, critical revision of the manuscript for important intellectual content, and approval of the final manuscript. Jonas Bybjerg-Grauholm: acquisition of data, critical revision of the manuscript for important intellectual content, and approval of the final manuscript. Preben B. Mortensen: acquisition of data, critical revision of the manuscript for important intellectual content, and approval of the final manuscript. Thomas M. Werge: acquisition of data, critical revision of the manuscript for important intellectual content, and approval of the final manuscript. Mads Melbye: interpretation of results, critical revision of the manuscript for important intellectual content, and approval of the final manuscript. David M. Hougaard: acquisition of data, critical revision of the manuscript for important intellectual content, and approval of the final manuscript.

\section{Funding}

The iPSYCH study was funded by The Lundbeck Foundation Initiative for Integrative Psychiatric Research (www.iPSYCH.au.dk). This research has been conducted using the Danish National Biobank resource, supported by the Novo Nordisk Foundation. Data handling and analysis on the GenomeDK HPC facility was supported by NIMH (1U01MH109514-01 to ADB). Highperformance computer capacity for handling and statistical analysis of iPSYCH data on the GenomeDK HPC facility was provided by the Center for Genomics and Personalized Medicine, Aarhus University, and Central Region Denmark, and Centre for Integrative Sequencing, iSEQ, Aarhus University (grant to ADB). Furthermore, the project was supported by grants from the Fonden til Lægevidenskabens Fremme and the Dagmar Marshalls Foundation. All are purely philanthropic foundations. The funders had no role in the design of the study, data collection, analysis, interpretation of data, or in writing the manuscript.

\section{Availability of data and materials}

The data that support the findings of this study are available from iPSYCH consortium, but restrictions apply to the availability of these data, which were used under license for the current study, and so are not publicly available. Data are however available from the authors upon reasonable request and with permission of the iPSYCH consortium.

\section{Declarations}

\section{Ethics approval and consent to participate}

The study was approved by the Scientific Ethics Committees of the Central Denmark Region (www.komite.rm.dk) (J.nr.: 1-10-72-287-12) and the Danish Data Protection Agency (www.datatilsynet.dk) (J.nr.: 2012-41-0110). The Danish Neonatal Screening Biobank approved the use of the DBS samples. Detailed information on governance and ethics in the iPSYCH cohort is available at the iPSYCH website (www.ipsych.au.dk).

\section{Consent for publication}

Not applicable as no personally identifiable data is available and results are presented as aggregated data.

\section{Competing interests}

The authors declare that they have no competing interests.

\section{Author details}

${ }^{1}$ Department of Epidemiology Research, Statens Serum Institut, Copenhagen, Denmark. ${ }^{2}$ Department of Neurosurgery, Copenhagen University Hospital, Blegdamsvej 9, DK-2100 Copenhagen, Denmark. ${ }^{3}$ Department of Clinical Medicine, University of Copenhagen, Copenhagen, Denmark. ${ }^{4}$ Department for Congenital Disorders, Statens Serum Institut, Copenhagen, Denmark. ${ }^{5}$ The Lundbeck Foundation Initiative for Integrative Psychiatric Research, iPSYCH, Aarhus, Denmark. ${ }^{6}$ Department of Biomedicine - Human Genetics, Aarhus University, Aarhus, Denmark. ${ }^{7}$ Bioinformatics Research Centre, Aarhus University, Aarhus, Denmark. ${ }^{8}$ Center for Genome Analysis and Personalized
Medicine, Aarhus, Denmark. ${ }^{9}$ Mental Health Centre, Capital Region of Denmark, Copenhagen, Denmark. ${ }^{10}$ Department of Biomedicine and the iSEQ Centre, Aarhus University, Aarhus, Denmark. ${ }^{11}$ Centre for Register Research, Institute of Economics, Aarhus University, Aarhus, Denmark. ${ }^{12}$ Department of Medicine, Stanford University School of Medicine, Stanford, CA, USA. ${ }^{13}$ Department of Biomedical Science, University of Copenhagen, Copenhagen, Denmark.

Received: 14 July 2020 Accepted: 15 April 2021

Published online: 28 April 2021

\section{References}

1. Parner ET, Thorsen P, Dixon G, de Klerk N, Leonard H, Nassar N, et al. A comparison of autism prevalence trends in Denmark and Western Australia. J Autism Dev Disord. 2011;41(12):1601-8. https://doi.org/10.1007/s10803011-1186-0.

2. Schendel DE, Thorsteinsson E. Cumulative incidence of autism into adulthood for birth cohorts in Denmark, 1980-2012. JAMA. 2018;320(17): 1811-3. https://doi.org/10.1001/jama.2018.11328.

3. Robinson S, Howlin P, Russell A. Personality traits, autobiographical memory and knowledge of self and others: a comparative study in young people with autism spectrum disorder. Autism. 2017;21(3):357-67. https://doi.org/1 $0.1177 / 1362361316645429$.

4. Lai MC, Lombardo MV, Baron-Cohen S. Autism. Lancet. 2014;383(9920):896910. https://doi.org/10.1016/S0140-6736(13)61539-1.

5. Leyfer OT, Folstein SE, Bacalman S, Davis NO, Dinh E, Morgan J, et al. Comorbid psychiatric disorders in children with autism: interview development and rates of disorders. J Autism Dev Disord. 2006;36(7):84961. https://doi.org/10.1007/s10803-006-0123-0.

6. Shen MD. Cerebrospinal fluid and the early brain development of autism. J Neurodev Disord. 2018;10(1):39. https://doi.org/10.1186/s11689-018-9256-7.

7. Shen MD, Kim SH, McKinstry RC, Gu H, Hazlett HC, Nordahl CW, et al. Increased extra-axial cerebrospinal fluid in high-risk infants who later develop autism. Biol Psychiatry. 2017:82(3):186-93. https://doi.org/10.1016/j. biopsych.2017.02.1095

8. Shen MD, Nordahl CW, Li DD, Lee A, Angkustsiri K, Emerson RW, et al. Extraaxial cerebrospinal fluid in high-risk and normal-risk children with autism aged 2-4 years: a case-control study. Lancet Psychiatry. 2018;5(11):895-904. https://doi.org/10.1016/S2215-0366(18)30294-3.

9. Shen MD, Nordahl CW, Young GS, Wootton-Gorges SL, Lee A, Liston SE, et al. Early brain enlargement and elevated extra-axial fluid in infants who develop autism spectrum disorder. Brain. 2013;136(Pt 9):2825-35. https://doi. org/10.1093/brain/awt166.

10. Schmidt LB, Corn G, Wohlfahrt J, Melbye M, Munch TN. School performance in children with infantile hydrocephalus: a nationwide cohort study. Clin Epidemiol. 2018;10:1721-31. https://doi.org/10.2147/CLEP.S178757.

11. Marino MA, Morabito R, Vinci S, Germano A, Briguglio M, Alafaci C, et al. Benign external hydrocephalus in infants. A single centre experience and literature review. Neuroradiol J. 2014;27(2):245-50. https://doi.org/10.15274/ NRJ-2014-10020.

12. Tucker J, Choudhary AK, Piatt J. Macrocephaly in infancy: benign enlargement of the subarachnoid spaces and subdural collections. J Neurosurg Pediatr. 2016;18(1):16-20. https://doi.org/10.3171/2015.12.PEDS15600.

13. Lindquist $B$, Carlsson G, Persson EK, Uvebrant P. Behavioural problems and autism in children with hydrocephalus: a population-based study. Eur Child Adolesc Psychiatry. 2006;15(4):214-9. https://doi.org/10.1007/s00787-006-0525-8.

14. Andersen TF, Madsen M, Jorgensen J, Mellemkjoer L, Olsen JH, The Danish National Hospital Register. A valuable source of data for modern health sciences. Dan Med Bull. 1999:46(3):263-8.

15. Norgaard-Pedersen B, Hougaard DM. Storage policies and use of the Danish Newborn Screening Biobank. J Inherit Metab Dis. 2007;30(4):530-6. https:// doi.org/10.1007/s10545-007-0631-x.

16. Mors O, Perto GP, Mortensen PB. The Danish Psychiatric Central Research Register. Scand J Public Health. 2011;39(7 Suppl):54-7. https://doi.org/10.11 77/1403494810395825.

17. Mainz J, Hess MH, Johnsen SP. The Danish unique personal identifier and the Danish Civil Registration System as a tool for research and quality improvement. Int J Qual Health Care. 2019;31(9):717-20. https://doi.org/10.1 093/intqhc/mzz008.

18. Pedersen CB, Bybjerg-Grauholm J, Pedersen MG, Grove J, Agerbo E, Baekvad-Hansen M, et al. The iPSYCH2012 case-cohort sample: new 
directions for unravelling genetic and environmental architectures of severe mental disorders. Mol Psychiatry. 2018;23(1):6-14. https://doi.org/10.1038/ mp.2017.196.

19. Lauritsen MB, Jorgensen M, Madsen KM, Lemcke S, Toft S, Grove J, et al. Validity of childhood autism in the Danish Psychiatric Central Register: findings from a cohort sample born 1990-1999. J Autism Dev Disord. 2010; 40(2):139-48. https://doi.org/10.1007/s10803-009-0818-0.

20. Guyot LL, Michael DB. Post-traumatic hydrocephalus. Neurol Res. 2000;22(1): 25-8. https://doi.org/10.1080/01616412.2000.11741034.

21. Moon SJ, Hwang JS, Shin AL, Kim JY, Bae SM, Sheehy-Knight J, et al. Accuracy of the Childhood Autism Rating Scale: a systematic review and meta-analysis. Dev Med Child Neurol. 2019;61(9):1030-8. https://doi.org/1 $0.1111 / \mathrm{dm} c \mathrm{n} .14246$

22. Anttila V, Bulik-Sullivan B, Finucane HK, Walters RK, Bras J, Duncan L, et al. Analysis of shared heritability in common disorders of the brain. Science. 2018;360:6395

23. Brett $\mathrm{D}$, Warnell $\mathrm{F}$, McConachie $\mathrm{H}$, Parr JR. Factors affecting age at ASD diagnosis in UK: no evidence that diagnosis age has decreased between 2004 and 2014. J Autism Dev Disord. 2016;46(6):1974-84. https://doi.org/1 0.1007/s10803-016-2716-6.

24. Levy SE, Giarelli E, Lee LC, Schieve LA, Kirby RS, Cunniff C, et al. Autism spectrum disorder and co-occurring developmental, psychiatric, and medical conditions among children in multiple populations of the United States. J Dev Behav Pediatr. 2010;31(4):267-75. https://doi.org/10.1097/DBP. 0b013e3181d5d03b.

25. Del Re EC, Bouix S, Fitzsimmons J, Blokland GAM, Mesholam-Gately R, Wojcik J, et al. Diffusion abnormalities in the corpus callosum in first episode schizophrenia: Associated with enlarged lateral ventricles and symptomatology. Psychiatry Res. 2019;277:45-51. https://doi.org/10.1016/j. psychres.2019.02.038.

26. Del Re EC, Konishi J, Bouix S, Blokland GA, Mesholam-Gately Rl, Goldstein J, et al. Enlarged lateral ventricles inversely correlate with reduced corpus callosum central volume in first episode schizophrenia: association with functional measures. Brain Imaging Behav. 2016;10(4):1264-73. https://doi. org/10.1007/s11682-015-9493-2.

27. Sowell ER, Levitt J, Thompson PM, Holmes CJ, Blanton RE, Kornsand DS, et al. Brain abnormalities in early-onset schizophrenia spectrum disorder observed with statistical parametric mapping of structural magnetic resonance images. Am J Psychiatry. 2000;157(9):1475-84. https://doi.org/1 0.1176/appi.ajp.157.9.1475.

28. Vanhala V, Junkkari A, Korhonen VE, Kurki Ml, Hiltunen M, Rauramaa T, et al. Prevalence of Schizophrenia in Idiopathic Normal Pressure Hydrocephalus. Neurosurgery. 2019;84(4):883-9. https://doi.org/10.1093/neuros/nyy147.

29. Yoshino Y, Yoshida T, Mori T, Hirota S, Iga J, Ueno S. Risk of idiopathic normal pressure hydrocephalus in older inpatients with schizophrenia. Int Psychogeriatr. 2016;28(5):863-8. https://doi.org/10.1017/S1041610215001763.

\section{Publisher's Note}

Springer Nature remains neutral with regard to jurisdictional claims in published maps and institutional affiliations.

Ready to submit your research? Choose BMC and benefit from:

- fast, convenient online submission

- thorough peer review by experienced researchers in your field

- rapid publication on acceptance

- support for research data, including large and complex data types

- gold Open Access which fosters wider collaboration and increased citations

- maximum visibility for your research: over $100 \mathrm{M}$ website views per year

At $\mathrm{BMC}$, research is always in progress.

Learn more biomedcentral.com/submissions 